\title{
ORIGINAL
}

\section{CAUSAS Y TENDENCIA DE LA MORTALIDAD EN UNA PRISIÓN ESPAÑOLA (1994-2009)}

\author{
Julio García-Guerrero, Enrique J Vera-Remartínez y Manuel V. Planelles Ramos.
}

Servicios Médicos. Centro Penitenciario de Castellón I.

\section{RESUMEN}

Fundamento: El conocimiento de las causas de mortalidad puede ser una herramienta valiosa para procurar mejores cuidados de salud a las personas encarceladas. El objetivo del trabajo es describir la mortalidad y su tendencia en nuestro centro.

Método: Estudio descriptivo de los fallecimientos ocurridos entre el $01 / 01 / 1994$ y $31 / 12 / 2009$. Se compararon mediante el test exacto de Fisher dos periodos de 8 años para valorar cambios en las causas de mortalidad. Previa estandarización indirecta de tasas, comparamos la mortalidad de nuestro centro con la del resto de las prisiones españolas. Mediante un modelo de regresión lineal establecimos la tendencia de las tasas de mortalidad.

Resultados: Hubo 59 defunciones, 58 en hombres, con una mediana de edad de 34,9 años $(28,7-40,4)$. El 64,4\% eran VIH+. Desde 1994 hasta 2001 la principal causa de mortalidad fue la infección VIH $(48,6 \%)$ y la segunda los eventos cardiovasculares $(10,8 \%)$, mientras que entre 2002 y 2009 los eventos cardiovasculares causaron un 31,8\% y la infección VIH un $22,7 \%(\mathrm{p}=0,026)$. Las tasas crudas de mortalidad anual tuvieron tendencia descendente a razón de 0,485 muertes internos/año (IC 95\%: 0,864 a -0,107). Estandarizando tasas corresponderían 42 muertes esperadas en todo el período, con una Razón de Mortalidad Estandarizada de 1,407 (IC 95\%: 1,071 a 1,816).

Conclusiones: La principal causa de mortalidad fue la enfermedad no-VIH, fundamentalmente por eventos cardiovasculares. La tendencia de la tasa de mortalidad fue descendente, aunque observamos un $40,7 \%$ más de defunciones de las que cabría esperar. neal.

Palabras clave: Mortalidad. Prisión. Tendencia. VIH. Regresión li-

Julio García Guerrero.

Centro Penitenciario de Castellón I.

Ctra de Alcora km 10.

12071 Castellón de la Plana.

garciaj@comcas.es

\section{ABSTRACT}

\section{Causes and Trends of mortality in a Spanish Prison (1994-2009)}

Background: There are a few mortality researches in prisons. To know this measure can to be important for take decisions of Public Health. The aim of the paper is to describe mortality and its trend in our prison.

Methods: This is a descriptive and retrospective study of the deaths between $01 / 01 / 1994$ and 31/12/2009. Two periods of 8 years have been compared through exact test of Fisher in order to detect changes in causes of mortality. First of all, we made an indirect standardization of rates and compare mortality in our institution with other Spanish prisons. Through linear regression model we have settled in trends of mortality rates.

Results: Had 59 deaths, 58 in men with a median age 34.9 years old (28.7- 40.4). 64,4\% were HIV+. From 1994 to 2001 the main cause of mortality was VIH infection (48.6\%) the second one was cardiovascular event (10.8\%), while that between 2002 and 2009 this trend have change, cardiovascular event caused (31.8\%) and VIH infection $(22.07 \%)$ $(\mathrm{p}=0.026)$. The annual crude mortality rate decreased -0.485 deaths inmates/year (IC 95\%: -0.864 to -0.107). Standardizing rates, we get 42 deaths expected for the wholly period, with Standarized Mortality Ratio of 1.407 (I.C. $95 \%: 1.071$ to 1.816 ).

Conclusions: The main cause of mortality has been no-HIV diseases, among these mainly cardiovascular events. The trend of mortality rate has been decreasing although we observe $40.7 \%$ plus of deaths than we would expect.

Key words: Mortality. Prison. Trend. VIH. Linear Regression. 


\section{INTRODUCCIÓN}

El conocimiento de las causas de mortalidad puede ser una herramienta valiosa para procurar mejores cuidados de salud a las personas encarceladas. Por ello, es deseable que los cambios de tendencia, si los hubiere, sean detectados precozmente para adoptar con prontitud medidas correctoras. En las prisiones hay una gran proporción de personas con déficits sanitarios, sobre todo debido a sus hábitos poco saludables y por otros factores de riesgo conductuales y sociales. Así, el abuso de substancias, las infecciones asociadas frecuentemente al uso de drogas -como la tuberculosis, la infección por el VIH o por el VHC-, y las enfermedades psiquiátricas, son más prevalentes en las personas encarceladas que en la población general ${ }^{1-4}$. La morbilidad debida a estos procesos puede hacer pensar que la mortalidad en los reclusos es superior a la de la población no presa, aspecto que ha sido observado en estudios con presos de España ${ }^{5}$ y con presos de fuera de nuestro país ${ }^{6,7}$, aunque también hay trabajos que discrepan de esta afirmación ${ }^{8-11}$, ya que los reclusos, por su frecuente edad joven, presentan también menor prevalencia de otros trastornos con alta morbi-mortalidad que la población no encarcelada. No obstante, el aumento en la edad de la población encarcelada puede estar contribuyendo a cambiar las causas clásicas de mortalidad en la población recluida, aproximándola a las causas de mortalidad en la población general.

Es posible además que estén influyendo otros factores en un posible cambio en la tasa de mortalidad de la población presa. Así, los tratamientos antirretrovirales, que supusieron en su momento una drástica reducción de la mortalidad en pacientes con infección $\mathrm{VIH}^{12}$, pueden estar modificado notablemente la mortalidad en los presos de España ${ }^{5,13}$.

Se desconoce cuál es la situación actual, ya que hay pocos trabajos publicados sobre este aspecto de la salud pública en las prisiones españolas.
El objetivo del trabajo es estudiar la mortalidad y sus causas en una prisión española e investigar sus cambios de tendencia.

\section{MATERIAL Y MÉTODO}

Se realizó un estudio descriptivo y retrospectivo en el centro penitenciario de Castellón I que abarcó el período entre 01-011994 y 31-12-2009. Para efectuar los cálculos se utilizó como denominador la población media del centro en cada uno de los 16 años del periodo estudiado. Las poblaciones medias anuales se obtuvieron hallando la media aritmética del número de internos presentes en el Centro el último día de cada mes.

La variable del estudio la constituyeron los fallecimientos ocurridos en el período estudiado. Se incluyeron todos los fallecimientos ocurridos bajo custodia, o lo que es lo mismo, en la prisión, en la unidad de ingreso de pacientes judiciales de nuestro hospital de referencia o en el traslado entre una y otra. No se incluyeron los fallecimientos ocurridos estando el interno en situación de permiso penitenciario o de libertad condicional.

Los datos fueron obtenidos a partir de fuentes secundarias: registro de defunciones del centro, historias clínicas de las personas internas fallecidas, boletines anuales de la Subdirección General de Sanidad Penitenciaria (SGSP), y los datos de población a partir de los registros de la oficina de régimen del establecimiento.

De todos los casos de fallecimiento se recopiló: fecha del óbito, con el día de la semana y estación del año, fecha de nacimiento, sexo, serología de VIH, en los casos $\mathrm{VIH}+$ se recogió también la cifra de linfocitos CD4+ más próxima al óbito (como máximo de 4 meses atrás) y la causa de muerte que figurase en el informe hospitalario de alta por defunción o en el informe forense en los casos en que se realizó autopsia. Todas las causas de muerte se codificaron según la clasificación internacional de enfermedades 
CIE-10 y posteriormente se clasificaron en 5 grupos: muertes relacionadas con la infección VIH, muertes por enfermedad no-VIH, suicidios, muertes por reacciones adversas a drogas y muertes accidentales.

Posteriormente se reclasificaron las muertes por enfermedad no-VIH como muertes por enfermedades cardiovasculares (ECV), enfermedades tumorales, enfermedades hepáticas, enfermedades circulatorias, enfermedades respiratorias, enfermedades infecciosas no VIH y otras.

Se registraron a partir de los Boletines Anuales de la SGSP las tasas específicas de mortalidad por año del conjunto de la población penitenciaria española. En analogía con estos Boletines, las tasas de mortalidad halladas se expresan en tantos por mil internos (\%) y año.

Los datos fueron procesados con el paquete informático SPSS v. 15.

Inicialmente se realizó un análisis descriptivo de las variables principales del estudio. Las cualitativas se expresaron en frecuencias absolutas y relativas, y las cuantitativas en medianas con sus percentiles (p25-p75). Se utilizó la determinación de Tukey. Se realizó una prueba de comparación de una distribución observada a una distribución teórica para establecer si había algún día concreto de la semana, o estación del año, estadísticamente significativo en el número de defunciones con respecto al resto de días o estaciones del año, mediante la utilización de una prueba no paramétrica como es el Test de $\chi 2$, valorando su significación estadística.

Se establecieron 2 periodos similares de tiempo de 8 años cada uno. Periodo 1: del 01-01-1994 al 31-12-2001; Periodo 2: del 01-01-2002 al 31-12-2009 y se comparó si habían existido diferencias entre las principales causas de mortalidad en cada uno de ellos. Se utilizó el test exacto de Fisher al existir alguna frecuencia inferior a $5 \mathrm{y}$ en las que encontramos diferencias, implementamos un modelo de regresión logística binaria para cuantificar la razón de ventajas (OR).

Posteriormente, mediante modelos de regresión lineal, considerando como variables dependientes las tasas de mortalidad del centro y las tasas de mortalidad de Instituciones Penitenciarias, y como variables independientes cada uno de los años, ajustando por la población media del centro y la población media de Instituciones Penitenciarias mediante el método de exclusión secuencial hacia atrás (Backward), apreciamos la tendencia experimentada por las distintas tasas de mortalidad.

Se realizó una comparación de la mortalidad en nuestro centro con respecto a la mortalidad de la población penitenciaria española, utilizando un proceso de estandarización indirecta de tasas por años obteniendo las muertes esperadas y se calculó la Razón de Mortalidad Estandarizada (SMR) con su intervalo de confianza (IC) al 95\%, empleando la macro !ISR v2003.07.15 para SPSS (C) JM. Doménech, A. Bonillo, R. Granero) ${ }^{14}$.

Consideraciones éticas: el diseño del estudio recibió la preceptiva autorización administrativa para su realización por la Secretaría General de Instituciones Penitenciarias del Ministerio del Interior del gobierno de España. Al tratarse de un estudio de consulta de datos de historias clínicas y siendo anónimos los datos, no se consideró necesario el concurso de un Comité Ético de Investigación Clínica.

\section{RESULTADOS}

La población media de la prisión en el período estudiado fue de 606 internos. Se produjeron 59 defunciones, 58 en hombres. La tasa cruda global de mortalidad fue de $6,182 \%$, oscilando entre $12,605 \%$ 
la máxima en 1997 y 1,757 en 2003 . La edad mediana fue de 34,9 años (28,7$39,8)$. El grupo etario más afectado fue el comprendido entre 31 y 40 años (26 muertes $-44,1 \%$-) (figura 1). 38 de los sujetos $(64,4 \%)$ eran VIH+ al fallecer, tenían una edad mediana de 34,1 años $(27,8-38,0)$ y presentaban una mediana de linfocitos CD4 de 200,5 cels/ $\mu 1$ (35-360).

La principal causa de mortalidad en todo el período fue la muerte por enfermedad no-VIH con 27 defunciones $(45,8 \%$ del total, tasa de $2,785 \%$ ), seguida de las muertes por VIH/Sida con 23 (39\% y tasa de $2,372 \%$ ), reacción adversa a drogas 5 $(8,5 \%$ y 0,516$)$, suicidios $3(5,1 \%$ y $0,309 \%)$ y accidentes/agresiones $1(1,7 \%$ y $0,103 \%$ ). Los sujetos VIH+ fallecieron fundamentalmente por sida $23(60,5 \%)$ y por ECV 5 (13,2\%), mientras que los noVIH lo hicieron por ECV $6(28,6 \%)$ y reacción adversa a drogas 4 (19\%) (tabla $1)$.
En la tabla 2 se muestran las muertes habidas según el período estudiado, con su tasa de mortalidad específica. Entre 1994 y 2001 la principal causa de mortalidad fue el VIH/Sida con 18 fallecimientos (48,6\% del total, tasa de 4,128\%o), mientras que entre 2002 y 2009 lo fueron los eventos cardiovasculares con $7(31,8 \%$, tasa de 1,310 ), siendo las diferencias estadísticamente significativas $(\mathrm{p}=0,026)$ (figura 2), lo que supone que actualmente hay 6 veces más probabilidades de fallecer en prisión por un ECV que por Sida (OR: 6,3; IC 95\% 1,3-30,5). La primavera fue la estación durante la que se produjeron más defunciones $(21 ; 35,6 \%)$ y durante la que menos fue el verano $(8 ; 13,6 \%)$, siendo las diferencias estadísticamente significativas $(p=0,048)$.

La estandarización de tasas muestra que corresponderían a nuestro centro 42 muertes esperadas en todo el período, con una Razón de Mortalidad Estandarizada

\section{Figura 1}

Distribución de las defunciones por edad $(n=59)$

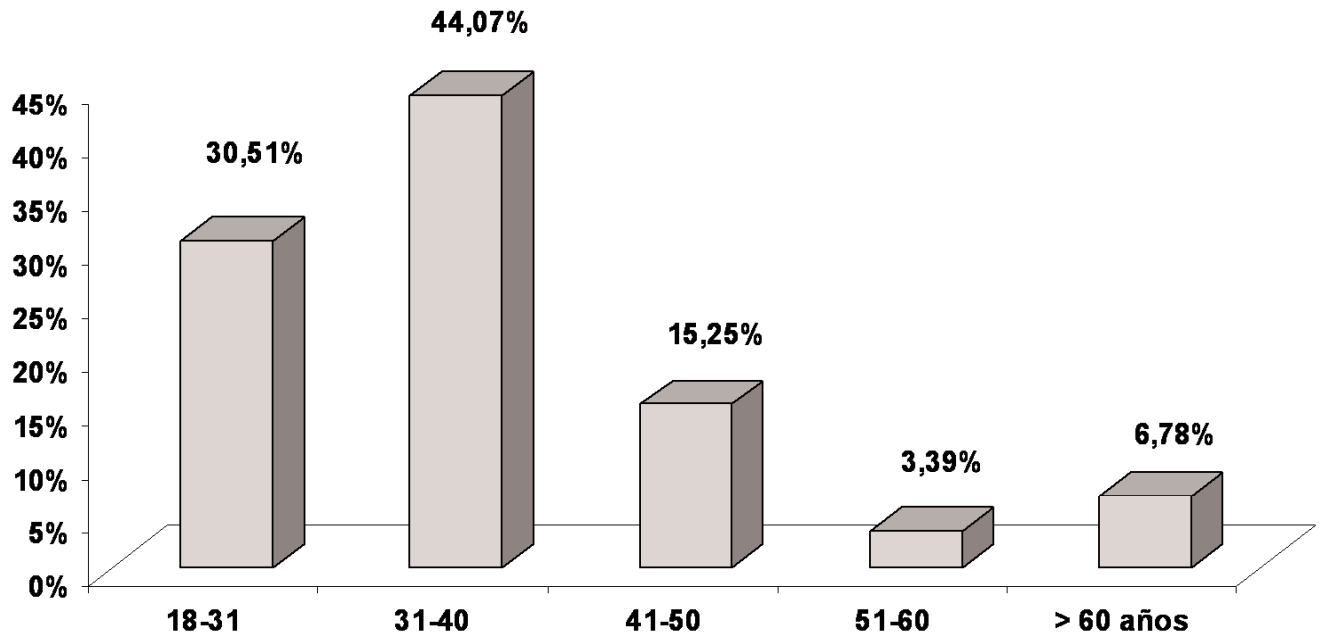




\section{Tabla 1}

\section{Principales causas de mortalidad según serología VIH}

\begin{tabular}{|c|c|c|c|c|}
\hline CAUSAS & $\begin{array}{c}\text { VIH } \\
\text { Positivo }\end{array}$ & $\begin{array}{c}\text { VIH } \\
\text { Negativo }\end{array}$ & Total & $\begin{array}{c}\mathrm{TME}_{\mathrm{p}} \\
1994-2009\end{array}$ \\
\hline Muertes por enfermedad no-VIH: & $14(36,8 \%)$ & $13(61,9 \%)$ & $27(45,8 \%)$ & $2,785 \%$ \\
\hline Cardiovasculares & $5(13,2 \%)$ & $6(28,6 \%)$ & $11(18,6 \%)$ & $1,134 \%$ \\
\hline Tumorales & $0(0,0 \%)$ & $3(14,3 \%)$ & $3(5,1 \%)$ & $0,309 \%$ \\
\hline Hepáticas & $3(7,9 \%)$ & $0(0,0 \%)$ & $3(5,1 \%)$ & $0,309 \%$ \\
\hline Respiratorias & $2(5,3 \%)$ & $1(4,8 \%)$ & $3(5,1 \%)$ & $0,309 \%$ \\
\hline Circulatorias & $1(2,6 \%)$ & $2(9,5 \%)$ & $3(5,1 \%)$ & $0,309 \%$ \\
\hline Infecciosas & $2(5,3 \%)$ & $0(0,0 \%)$ & $2(3,4 \%)$ & $0,206 \%$ \\
\hline Otras & $1(2,6 \%)$ & $1(4,8 \%)$ & $2(3,4 \%)$ & $0,206 \%$ \\
\hline Muertes por VIH / SIDA & $23(60,5 \%)$ & $0(0,0 \%)$ & $23(39,0 \%)$ & $2,372 \%$ \\
\hline Muertes por reacciones adversas a drogas & $1(2,6 \%)$ & $4(19,0 \%)$ & $5(8,5 \%)$ & $0,516 \%$ \\
\hline Muertes por suicidio & $0(0,0 \%)$ & $3(14,3 \%)$ & $3(5,1 \%)$ & $0,309 \%$ \\
\hline Muertes ocurridas de forma accidental & $0(0,0 \%)$ & $1(4,8 \%)$ & $1(1,7 \%)$ & $0,103 \%$ \\
\hline TOTALES & $38(100 \%)$ & $21(100 \%)$ & $59(100 \%)$ & $6,183 \%$ \\
\hline
\end{tabular}

a TMEP $=$ Tasa de Mortalidad Específica del periodo estudiado por cada 1000 internos y año.

Tabla 2

Principales causas de mortalidad por periodos

\begin{tabular}{|c|c|c|c|c|}
\hline CAUSAS: & $\begin{array}{l}\text { Periodo 1: } \\
\text { 1994-2001 }\end{array}$ & $\begin{array}{l}\text { Periodo 2: } \\
2002-2009\end{array}$ & $\begin{array}{c}{ }^{2} \text { TMEp } \\
1994-2001\end{array}$ & $\begin{array}{c}{ }^{2} \text { TMEP } \\
2002-2009\end{array}$ \\
\hline Muertes por enfermedad no-VIH: & $13(35,1 \%)$ & $14(63,6 \%)$ & $2,982 \%$ & $2,620 \%$ \\
\hline Cardiovasculares & $4(10,8 \%)$ & $7(31,8 \%)$ & $0,917 \%$ & $1,310 \%$ \\
\hline Tumorales & $1(2,7 \%)$ & $2(9,1 \%)$ & $0,229 \%$ & $0,374 \%$ \\
\hline Hepáticas & $3(8,1 \%)$ & $0(0,0 \%)$ & $0,688 \%$ & $0,0 \%$ \\
\hline Respiratorias & $1(2,7 \%)$ & $2(9,1 \%)$ & $0,229 \%$ & $0,374 \%$ \\
\hline Circulatorias & $1(2,7 \%)$ & $2(9,1 \%)$ & $0,229 \%$ & $0,374 \%$ \\
\hline Infecciosas & $2(5,4 \%)$ & $0(0,0 \%)$ & $0,459 \%$ & $0,0 \%$ \\
\hline Otras & $1(2,7 \%)$ & $1(4,5 \%)$ & $0,229 \%$ & $0,187 \%$ \\
\hline Muertes por VIH / SIDA & $18(48,6 \%)$ & $5(22,7 \%)$ & $4,128 \%$ & $0,936 \%$ \\
\hline Muertes por reacción adversa a drogas & $2(5,4 \%)$ & $3(13,6 \%)$ & $0,459 \%$ & $0,561 \%$ \\
\hline Muertes por suicidio & $3(8,1 \%)$ & $0(0,0 \%)$ & $0,688 \%$ & $0,0 \%$ \\
\hline Muertes ocurridas de forma accidental & $1(2,7 \%)$ & $0(0,0 \%)$ & $0,229 \%$ & $0,0 \%$ \\
\hline $\begin{array}{l}\text { TOTALES: } \\
\end{array}$ & $37(100 \%)$ & $22(100 \%)$ & $8,486 \%$ & $4,117 \%$ \\
\hline
\end{tabular}

a TMEP $=$ Tasa de Mortalidad Específica del periodo estudiado por cada 1000 internos y año.

de 1,407 ( IC 95\%: 1,071 a 1,816) (tabla 3 ), por lo que se han producido un $40,7 \%$ más de defunciones si se hubiesen aplicado las mismas tasas que para la población penitenciaria española.

A pesar de estos datos se objetivó una tendencia claramente descendente a razón de -0,485 muertes internos/año (IC 95\%:-0,864 a -0,107; $\mathrm{p}=0,016)$, siendo esta tendencia mucho más acusada que en el conjunto de las prisiones españolas, cuya tendencia es igualmente descendente pero a razón de $-0,210$ muertes internos/año (IC 95\%:-0,327 a -0,093; $\mathrm{p}=0,002)($ figura 3$)$. 


\section{Figura 2}

\section{Principales causas de mortalidad por períodos}

18

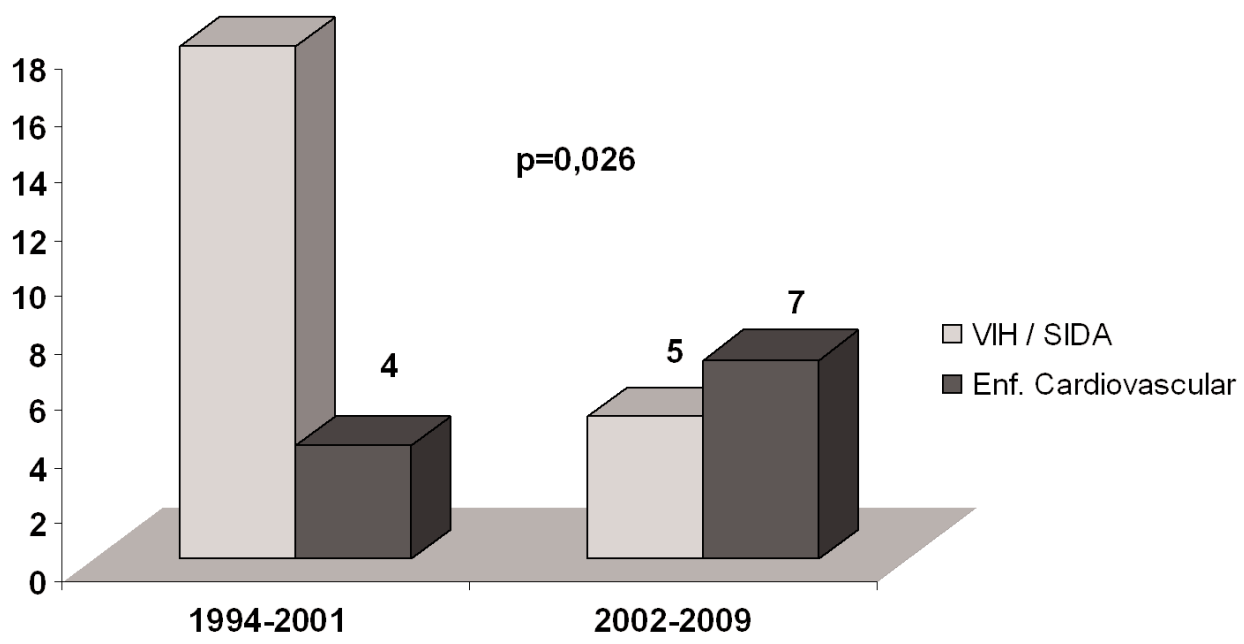

Tabla 3

Estandarización de tasas

\begin{tabular}{|c|c|c|c|c|c|}
\hline \multicolumn{4}{|c|}{ Defunciones observadas } & \multicolumn{2}{|c|}{ Defunciones esperadas } \\
\hline Estrato: & $\begin{array}{c}\text { Personas } \\
\text { Tiempo: }\end{array}$ & Casos & ${ }^{2} \mathrm{TMc}$ & Casos & ${ }^{\mathrm{b}} \mathrm{TMe}$ \\
\hline 1994 & 662 & 8 & 12,084 & 5,142 & 7,776 \\
\hline 1995 & 622 & 6 & 9,646 & 4,485 & 7,209 \\
\hline 1996 & 565 & 5 & 8,849 & 3,327 & 5,889 \\
\hline 1997 & 476 & 6 & 12,605 & 2,193 & 4,607 \\
\hline 1998 & 487 & 3 & 6,160 & 1,842 & 3,781 \\
\hline 1999 & 502 & 4 & 7,968 & 2,070 & 4,123 \\
\hline 2000 & 513 & 1 & 1,949 & 1,611 & 3,140 \\
\hline 2001 & 531 & 5 & 9,416 & 1,886 & 3,550 \\
\hline 2002 & 567 & 1 & 1,763 & 1,973 & 3,480 \\
\hline 2003 & 569 & 1 & 1,757 & 1,939 & 3,407 \\
\hline 2004 & 608 & 2 & 3,289 & 2,156 & 3,546 \\
\hline 2005 & 596 & 3 & 5,033 & 2,285 & 3,834 \\
\hline 2006 & 664 & 2 & 3,012 & 2,659 & 4,004 \\
\hline 2007 & 775 & 2 & 2,580 & 2,770 & 3,573 \\
\hline 2008 & 781 & 8 & 10,243 & 2,897 & 3,708 \\
\hline 2009 & 781 & 2 & 2,5608 & 2,698 & 3,4548 \\
\hline TOTAL & 9699 & 59 & 6,182 & 41,933 & 4,223 \\
\hline \multicolumn{6}{|c|}{${ }^{\mathrm{e}} \mathrm{SMR}(\mathrm{O} / \mathrm{E})=1,407 ;$ IC $95 \%(1,070$ a 1,815$)$} \\
\hline
\end{tabular}


Figura 3

Tendencias de las tasas de mortalidad

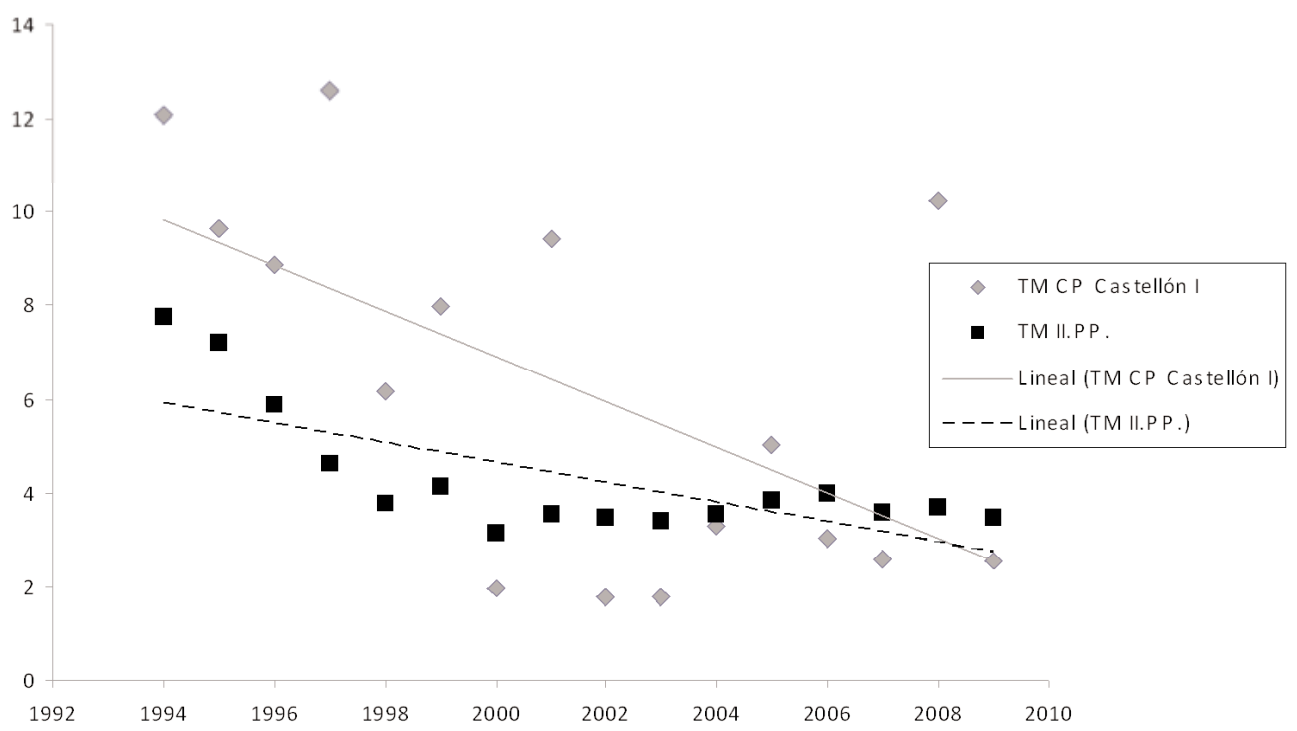

\section{DISCUSIÓN}

Hemos estudiado la evolución y tendencia de las tasas de mortalidad y sus causas en una prisión española de tamaño medio en un largo espacio de tiempo. La tasa cruda global que encontramos es inferior a la tasa de mortalidad de la población general española para el año 2009 , cifrada en $8,343 \%{ }^{15}$, a diferencia de lo observado en una investigación anterior sobre el mismo asunto, en la que esta tasa era superior en prisión ${ }^{5}$. Esto se ha logrado a costa de objetivar un descenso profundo y continuado de la tasa de mortalidad de nuestro centro que, no obstante, es inferior al que encontramos en nuestro anterior trabajo ${ }^{5}$. Probablemente haya que achacar este fenómeno a que en el primero se recogió el grueso del descenso de mortalidad por sida, producida a raíz de la disponibilidad de los nuevos y potentes fármacos antirretrovirales a finales de 1996. Encontramos una tasa global de mortalidad mayor que la del conjunto de la población penitenciaria española para todo el período estudiado, pero hubo un mayor descenso en nuestras tasas que en el conjunto del sistema penitenciario español, el cual ha mantenido una tendencia a la estabilidad con ligeros ascensos en los últimos diez años ${ }^{16}$. Esa tendencia estable o ligeramente creciente de las muertes ocurridas bajo custodia también se aprecia en el sistema penal norteamericano y en ambos casos se debe fundamentalmente al aumento de las muertes por enfermedad no-sida, ya que en ambos sistemas se ha producido un acusado descenso de las muertes por sida ${ }^{16-18}$.

La infección VIH/Sida ha marcado la actividad sanitaria en las prisiones españolas en los últimos 25 años. Hubo momentos en que la tercera parte de las personas que ingresaban en prisión lo hacían infectados por el VIH ${ }^{19}$ y un estudio que abarcó la totalidad de las personas encarceladas en 1989 cifraba su prevalencia en el $28,4 \%$ de la población reclusa ${ }^{20}$. Además, hasta el año 2000 el sida era la principal causa de mortalidad en prisión ${ }^{21}$. En nuestro trabajo la 
infección VIH/Sida es la segunda causa de muerte global, aunque es la primera en el tramo inicial de los dos en que dividimos el período a estudiar. La disponibilidad de fármacos antirretrovirales ha hecho que la mortalidad por VIH represente hoy menos del $10 \%$ de todas las muertes en prisión ${ }^{16} \mathrm{y}$ que en los últimos tres años no haya habido muertes por esta causa en nuestro centro, lo que puede contribuir a explicar ese descenso más acusado en nuestras tasas que el del conjunto de la población penitenciaria española. En cualquier caso, la magnitud del VIH en prisión es un fenómeno casi exclusivamente español y condicionado por el hábito de uso de drogas intravenosas (UDI). No ocurre lo mismo en el sistema penal americano en su conjunto, donde las muertes por sida son inferiores al $6 \%$ del total de los fallecimientos ${ }^{17}$, aunque puntualmente sobrepasen esta cifra hasta llegar al $9 \%{ }^{8}$; y en prisiones canadienses ${ }^{7}$ o inglesas $^{9}$ su baja magnitud hace que no merezcan mención específica como causa de muerte .

Hemos observado un aumento de las muertes por enfermedad común no-VIH, fundamentalmente a expensas de los eventos cardiovasculares. Pueden apuntarse tres razones para explicar este fenómeno: el aumento de edad general de los internos, la inusitada prevalencia de factores de riesgo cardiovascular en una población relativamente joven como es la nuestra ${ }^{22,23}$ y la alta proporción de consumidores de drogas, especialmente cocaína, y su consumo simultáneo con tabaco. Los efectos de la cocaína sobre la salud cardiovascular de adultos jóvenes hace tiempo que están bien establecidos $^{24}$. Es destacable la poca mortalidad aportada por la hepatitis C (3 casos), a diferencia de lo citado en un informe que habla de que esta enfermedad es responsable del $9 \%$ de las muertes ${ }^{25}$, lo que creemos que puede deberse al largo tiempo evolutivo de esta enfermedad y a que desde 2003 se oferta tratamiento con interferón pegilado más ribavirina a los pacientes que lo precisan.
Nuestras tasas de suicidio son algo menores que las del conjunto de la población penitenciaria española ${ }^{16}$, pero prácticamente doblan a las que se comunican para las prisiones norteamericanas ${ }^{18,26}$ y también son superiores a las de la población general española ${ }^{15}$, como lo son las de las prisiones norteamericanas ${ }^{26}$ e inglesas ${ }^{27}$. Estos datos hay que manejarlos con prudencia por el pequeño tamaño de la muestra estudiada, pero resaltamos que todos los suicidios se produjeron en el primer período de los estudiados. El mantenimiento y la aplicación estricta de un programa de prevención de suicidios, con especial énfasis en sujetos con trastornos psiquiátricos constatados, se ha demostrado efectivo para reducir los suicidios en prisiones ${ }^{8}$. Es probable que lo hecho en nuestra prisión en ese sentido tenga mucho que ver con este fenómeno.

Las muertes por reacción adversa a drogas es una frecuente fuente de mortalidad en prisión ${ }^{28}$, oscilando su rango entre el $17,7 \%$ y el $6,7 \%{ }^{8,28}$ de todas las producidas. Nuestros datos globales están en esos límites, pero en el segundo período se produjeron más muertes que en el primero. Ha habido un cambio en los últimos años en las intoxicaciones por drogas en las prisiones que tiene repercusiones en los resultados a la hora de combatirlas. Ya no se trata de la clásica sobredosis de heroína administrada en grupo, hay indicios que apuntan a que nos enfrentamos a intoxicaciones complejas y causadas por drogas de varias familias (benzodiacepinas, cocaína, neurolépticos atípicos, metadona $)^{16}$, administradas en solitario a primera hora de la noche, por lo que resulta muy difícil de combatir.

Cabe preguntarse por las razones más generales que hacen que la mortalidad descienda en prisión dada la gran morbilidad que se da entre los presos. Primeramente puede apuntarse un estilo de vida más disciplinado en prisión, con disminución de los comportamientos de riesgo que se dan en libertad. También los presos hacen más uso 
de los servicios médicos que estando en libertad, lo que facilita la detección temprana de problemas de salud que de otra forma podrían pasar desapercibidos. También es posible que condenas largas tengan que ver con ello, ya que un estudio francés relacionó un mayor tiempo de estancia en prisión con una menor mortalidad durante el internamiento ${ }^{10}$.

La principal limitación de nuestro trabajo es el tamaño de la muestra, que condiciona el análisis estadístico de los datos. También hay que ser muy cauteloso con el tamaño poblacional utilizado como denominador en los cálculos, debido a la gran movilidad de los presos. Como forma de minimizar este riesgo utilizamos el valor promedio de los presentes el último día de cada mes de los años estudiados. Por último, hemos de mencionar aquí que el estudio está hecho en una sola prisión y es difícil extrapolar los hallazgos a otras o al conjunto del sistema, incluso se pueden obtener resultados diferentes, como es nuestro caso.

Por último, se pueden apuntar dos sesgos que pueden ser causa de alteración de los resultados. El primero es no tener en cuenta los fallecimientos producidos fuera de la prisión, en aplicación del artículo 196.2 del Reglamento Penitenciario que prevé el adelantamiento de la libertad condicional por motivos humanitarios, fundamentalmente por enfermedad irreversible y terminal. Si esto no se hiciera sistemáticamente es probable que nuestras tasas de mortalidad fueran mayores, sobre todo para la patología tumoral y la enfermedad terminal hepática. El segundo sesgo se produce antes de entrar en prisión: posiblemente los ciudadanos menos sanos tienen menos posibilidades de cometer delitos y por tanto de ser condenados a penas de prisión o, en caso de ser condenados, tienen más posibilidades de ser condenados a penas que no llevan aparejado el encarcelamiento. Además, entendemos que hay una causa más de fondo: la morbilidad en las prisiones es, como deci- mos, alta y debida a procesos graves pero muy específicos (VIH, VHC, patología psiquiátrica, tuberculosis), no obstante su mortalidad ha disminuido por todas las razones apuntadas más arriba. Por la edad media de los presos (alrededor de cuarenta años), su tasa de mortalidad probablemente debería ser menor que la de la población general. Es lógico que a una población más joven le corresponda una menor tasa de mortalidad y lo que probablemente esté ocurriendo es que una situación que se distorsionó con la aparición del consumo de drogas y las enfermedades asociadas a él en las prisiones a lo largo de la década de los ochenta del siglo pasado, como la enfermedad por VIH, esté retrocediendo.

Como corolario decir que presentamos un trabajo sobre un aspecto de salud pública poco conocido en España, como es la mortalidad entre las personas privadas de libertad. Objetivamos un cambio en las causas de mortalidad en los últimos 16 años. La infección VIH/Sida ha dejado de ser la primera causa de muerte en prisión, y ahora son las enfermedades cardiovasculares. Urge implementar en prisión programas específicos de atención en estas patologías y de formación de los profesionales en los últimos avances en estas y otras patologías clásicas de atención primaria, como diabetes o hipertensión. De esta forma conseguiremos cumplir el papel fundamental que tienen los cuidados de salud en la prisión: proporcionar una atención médica de calidad a los miembros más vulnerables de nuestra sociedad y conseguir así también mejorar la Salud Pública general.

\section{BIBLIOGRAFÍA}

1.- Hammett TM, Harmon MP, Rhodes W. The burden of infectious disease among inmates of and releases from US correctional facilities, 1997. Am J Public Health. 2002; 92:1789-94.

2.- Wu ZH, Baillargeon J, Grady JJ, Black SA, Dunn K. HIV seroprevalence among newly incarcerated inmates in the Texas correctional system. Ann Epidemiol. 2001; 11: 342-6. 
3.- Fazel S, Baillargeon J. The health of prisoners. Lancet. 2011;377:956-65.

4.- García-Guerrero J, Marco-Mouriño A, Sáiz de la Hoya P, Vera-Remartínez EJ y Grupo de estudio PREVALHEP de prisiones. Estudio multicéntrico de prevalencia de infección tuberculosa latente entre los internados en las prisiones españolas. Rev Esp Sanid Penit. 2010; 12: 79-85.

5.- Vera-Remartínez EJ, Planelles-Ramos MV, GarcíaGuerrero J. Tendencia de la tasa de mortalidad en una prisión española (1994-2004). Rev Esp Salud Pública. 2005; 79: 673-82.

6.- Kariminia A, Law MG, Butler TG, Corben SP, Levy MH, Kaldor JM et al. Factors Associated with mortality in a cohort of Australian prisoners. Eur J Epidemiol. 2007; 22: 417-28.

7.- Wobeser WL, Datema J, Bechard B, Ford P. Causes of death among people in custody in Ontario, 19901999. CMAJ. 2002; 167: 1109-13

8.- Kim S, Ting A, Puisis M, Rodríguez S, Benson M, Mennela C et al. Deaths in the Cook County Jail: 10year report 1995-2004. J Urban Health. 2007; 84: 7084.

9.- Fazel S, Benning R. Natural deaths in male prisons: a 20-year mortality study. Eur J Public Health. 2006; 16: 441-4

10.- Clavel F, Benhamou S, Flamant R. Decreased mortality among male prisoners. Lancet. 1987: 10124.

11.- Novick L, Remmlinger E. A study of 128 deaths in New York City Correctional Facilities (1971-1976). Med Care. $1978 ; 16: 749-56$.

12.- Palella F, Delaney KM, Moorman AC, Loveless MO, Fhurer J, Satten GA et al. Declining morbidity and mortality among patients with advanced human inmunodeficency virus infection. N Engl J Med. 1998; 338: 853-60.

13.- García-Guerrero J, Vera-Remartínez EJ, González-Morán F. Morbimortalidad hospitalaria en presos VIH+: 10 años de cambios. Rev Esp Sanid Penit. 2005; 7: 14-22.

14.- Delgado M, Llorca J, Doménech JM. Investigación científica: fundamentos metodológicos y estadísticos. Barcelona: Signo; 2005. p.220-23.

15.- Instituto Nacional de Estadística. Indicadores demográficos básicos. Tasa Bruta de Mortalidad cal- culada para el año 2009. Disponible en: http://www.ine.es/jaxi/tabla.do?per=12\&type $=\mathrm{db} \& \mathrm{di}$ $\mathrm{vi}=\mathrm{IDB} \& \mathrm{idtab}=32$. (Citado el 28/12/2010).

16.- Secretaría General de Instituciones Penitenciarias. Coordinación de Sanidad Penitenciaria. Área de Salud Pública. Mortalidad en Instituciones Penitenciarias 2009. Madrid: Secretaría General de Instituciones Penitenciarias; 2010.

17.- Mumola CJ. U.S. Department of Justice. Bureau of Justice Statistics. Special Report: Suicide and Homicide in State Prisons and Local Jails. August 2005 . NCJ 210036 . Disponible en: www.bjs.ojp.usdoj.gov/content/pub/pdf/shsplj.pdf (Citado el 14-12-2010).

18.- Nooman ME. U.S. Departament of Justice. Bureau of Justice Statistics. Deaths in custody: State prison, Deaths 2001-2007. Statistical Tables. October 2010. D is p on ib le e $n$ : www.bjs.ojp.usdoj.gov/index.cfm?ty=pbdetail\&iid $=2$ 093. (Citado el 14-12-2010).

19.- Martín V, Bayas JM, Laliga A, Pumarola T, Vidal J, Jiménez de Anta MT et al. Epidemiology of HIV-1 infection in a Catalonian penitentiary. AIDS. 1990; 4:1023-6.

20.- Martín-Sánchez M. Programa de prevención y control de enfermedades transmisibles en Instituciones Penitenciarias. Rev Estudios Penit. 1990; Monográfico de Sanidad Penitenciaria 1: 51-67.

21.- Dirección General de Instituciones Penitenciarias. Subdirección General de Sanidad Penitenciaria. Área de Salud Pública. Mortalidad en Instituciones Penitenciarias 2000. Madrid: Dirección General de Instituciones Penitenciarias; 2001.

22.- Gimeno S, Esteban A, Andrés MA, Letamendi R, Llopis F. Estudio de la prevalencia de factores de riesgo cardiovascular en la población de un Centro Penitenciario tipo. Rev Esp Sanid Penit. 2010; 12(3suppl): 174.

23.- Mínguez C, Vera-Remartínez EJ, García-Guerrero J. Factores de riesgo vascular en pacientes infectados por el VIH en un centro penitenciario. Rev Clin Esp. 2011; 211: 9-16.

24.- Hollander JE. Cocaine-associated myocardial infarction. J R Soc Med. 1996; 89: 443-7.

25.- Harzke AJ, Baillargeon JG, Kelley MF, Diamond PM, Goodman KJ, Paar DP. HCV-related mortality among male prison inmates in Texas 1994-2003. Ann Epidemiol. 2009; 19: 582-9. 
26.- Baillargeon J, Penn JV, Thomas CR, Temple JR, Baillargeon G, Murray OJ. Psychiatric Disorders and Suicide in the Nation's Largest State Prison System. J Am Acad Psychiatry Law. 2009; 37: 188-93.

27.- Fazel S, Benning R, Danesh J. Suicides in male prisoners in England and Walles, 1978-2003. Lancet. 2005; 366: 1301-2.

28.- Wakeman SE, Bowman SE, Mckenzie M, Jeronimo A, Rich JD. Preventing Death among the Recently Incarcerated: An Argument for Naloxone Prescription Before Release. J Addict Dis. 2009; 28: 124-29. 